\title{
GROUP-VALUED CHARGES: COMMON EXTENSIONS AND THE INFINITE CHINESE REMAINDER PROPERTY
}

\author{
K. P. S. BHASKARA RAO AND R. M. SHORTT
}

(Communicated by Andrew M. Bruckner)

\begin{abstract}
We exhibit two consistent, integer-valued charges (finitely additive measures) which do not have a common, integer-valued extension. More generally, after introducing the notion of an infinitary Chinese remainder property for Abelian groups, we show that if a group has the common extension property, then the group must have the infinite Chinese remainder property. The class of groups with the common extension property is characterised as coincident with the class of cotorsion groups.
\end{abstract}

\section{INTRODUCTION}

We are concerned with finitely additive measures ("charges") taking values in a group $G$. All groups will be assumed Abelian, and we shall employ the usual additive notation for these groups, writing, for example, $n x-y$ for $x^{n} y^{-1}$ and indicating the neutral element of $\mathrm{G}$ with 0 . Let $X$ be a nonempty set, and let $\mathscr{A}$ be a field of subsets of $X$. A function $\mu: \mathscr{A} \rightarrow G$ is a ( $G$-valued) charge if $\mu(\varnothing)=0$ and $\mu\left(A_{1} \cup A_{2}\right)=\mu\left(A_{1}\right)+\mu\left(A_{2}\right)$ whenever $A_{1}$ and $A_{2}$ are disjoint sets in $\mathscr{A}$.

Now suppose that $\mathscr{A}$ and $\mathscr{B}$ are fields of subsets of $X$ and that $\mu: \mathscr{A} \rightarrow G$ and $\nu: \mathscr{B} \rightarrow G$ are $G$-valued charges. We say that $\mu$ and $\nu$ are consistent if $\mu(C)=\nu(C)$ whenever $C \in \mathscr{A} \cap \mathscr{B}$. For a given $G$, we are interested in whether any two consistent charges $\mu$ and $\nu$ have a common extension, i.e. whether there is a charge $\rho$ such that $\rho(A)=\mu(A)$ if $A \in \mathscr{A}$ and $\rho(B)=\nu(B)$ if $B \in \mathscr{B}$. The charge $\rho$ is to be defined on $\mathscr{A} \vee \mathscr{B}$, the field generated by $\mathscr{A} \cup \mathscr{B}$.

Say that a group $G$ has the common extension property if every pair of consistent $G$-valued charges has a common extension. It is known that the group $\mathbf{R}$ of real numbers has the extension property. In [1], it was shown that every algebraically compact group has this property. In [2], the authors exhibited an example (inspired by G. Bergman) of a group $G$ without the extension

Received by the editors May 7, 1990.

1980 Mathematics Subject Classification (1985 Revision). Primary 28B10; Secondary 20K99.

Key words and phrases. Charge, Chinese remainder theorem, cotorsion group, algebraically compact group. 
property. It will follow from results in this paper that the group $\mathbf{Z}$ of integers does not have the common extension property. In $\S 3$, we shall obtain a complete characterisation of groups with the common extension property.

The problem of extending a single charge to a larger field of sets has already been solved by Carlson and Prikry [3]. They proved the following result. Let $\mathscr{P}(X)$ be the power set field of $X$.

Lemma. Let $\mu: \mathscr{A} \rightarrow G$ be a $G$-valued charge on $(X, \mathscr{A})$. There is a charge $\rho: \mathscr{P}(X) \rightarrow G$ such that $\rho(A)=\mu(A)$ for each $A \in \mathscr{A}$

Tacit use will be made of this lemma at several points in the sequel.

Let $a$ and $b$ be elements of an Abelian group $G$ and let $n$ be a positive integer. Write $a \equiv b(\bmod n)$ if $n$ divides $a-b$, i.e. there is some $c \in G$ such that $a-b=n c$.

Chinese Remainder Theorem 0.2. Let $G$ be an Abelian group and suppose that $a_{1}, a_{2}, \ldots, a_{m}$ are elements of $G$. Let $n_{1}, n_{2}, \ldots, n_{m}$ be pairwise relatively prime, positive integers. Then there is some $x \in G$ such that $x \equiv a_{i}\left(\bmod n_{i}\right)$ for each $i=1,2, \ldots, m$.

Indication. The result is well known for $G=\mathbf{Z}$, and the usual proof, e.g. the one in [5], carries over to arbitrary Abelian groups.

We say that a group $G$ has the Chinese remainder property if, whenever $n_{1}, n_{2}, n_{3}, \ldots$ is a sequence of pairwise relatively prime, positive integers, and $a_{1}, a_{2}, a_{3}, \ldots$ are elements of $G$, then there is some $x \in G$ such that $x \equiv a_{i}\left(\bmod n_{i}\right)$ for each $i=1,2,3, \ldots$

An Abelian group $G$ is said to be algebraically compact if it is a direct summand in some compact (Hausdorff) topological group. Purely algebraic formulations of this concept are available. See Fuchs $[4, \S 38]$ for details.

We record a few observations about these ideas. Recall that a group $G$ is divisible if for each $a \in G$ and $n \in \mathbf{Z}$, there is some $b \in G$ with $a=n b$.

0.3. Every divisible group is algebraically compact. [4, §38]. In particular, the groups $\mathbf{Q}$ and $\mathbf{R}$ are algebraically compact, along with every vector space with scalars in these groups.

0.4. Finite groups are algebraically compact. (They are compact in the discrete topology.)

0.5 . Every algebraically compact group has the common extension property. This was established by A. Basile and K.P.S. Bhaskara Rao [1].

0.6. Every algebraically compact group has the Chinese remainder property. This can be established directly using the techniques in $\S 38$ in [4], but it will follow as a consequence of Theorem 2.1 infra. 
0.7. If $G$ has the Chinese remainder property and $\phi: G \rightarrow H$ is a homomorphism of $G$ onto a group $H$, then $H$ has the Chinese remainder property. (This is easily verified.)

0.8. The integers $\mathbf{Z}$ do not have the Chinese remainder property. By 0.7 , no nontrivial free Abelian group can have this property.

We close the preliminaries with a few remarks concerning $G$-valued integration. Given a field $\mathscr{A}$ of subsets of $X$, let $S(X, \mathscr{A})$ be the set of all functions $f: X \rightarrow \mathbf{Z}$ such that range $(f)$ is finite and such that $f^{-1}(n) \in \mathscr{A}$ for each $n \in \mathbf{Z}$. We see that $S(X, \mathscr{A})$ becomes a ring under pointwise addition and multiplication of functions.

Let $\mu: \mathscr{A} \rightarrow G$ be a $G$-valued charge. Given $A \in \mathscr{A}$, let $I_{A}$ be its indicator function. Then the mapping $I_{A} \rightarrow \mu(A)$ extends uniquely to a group homomorphism from $S(X, \mathscr{A})$ to $G$. The value of this homomorphism at $f \in S(X, \mathscr{A})$ we denote by $\int f d \mu$, the integral of $f$ with respect to $\mu$. If $A \in \mathscr{A}$, then, as usual, take

$$
\int_{A} f d \mu=\int f \cdot I_{A} d \mu
$$

\section{INDEPENDENT FIELDS}

Let $\mathscr{A}$ and $\mathscr{B}$ be fields of subsets, of a set $X$. Then $\mathscr{A}$ and $\mathscr{B}$ are said to be independent if $A \cap B \neq \varnothing$ whenever $A \in \mathscr{A}$ and $B \in \mathscr{B}$ are nonempty sets. Note that if $\mathscr{A}$ and $\mathscr{B}$ are independent, then $\mathscr{A} \cap \mathscr{B}=$ $\{\varnothing, X\}$. Given a sequence $\mathscr{C}_{1}, \mathscr{C}_{2}, \mathscr{C}_{3}, \ldots$ of fields of subsets of $X$, we call the sequence $\left(\mathscr{C}_{k}\right)$ jointly independent if for any finite subset of positive integers $k_{1}, k_{2}, k_{3}, \ldots, k_{n}$ and any nonvoid sets $C_{1}, C_{2}, C_{3}, \ldots, C_{n}$ in $\mathscr{C}_{k_{1}}, \mathscr{C}_{k_{2}}, \mathscr{C}_{k_{3}}, \ldots, \mathscr{C}_{k_{n}}$ respectively, we have $C_{1} \cap C_{2} \cap \cdots \cap C_{n} \neq \varnothing$.

Lemma 1.1. Let $\mathscr{C}_{1}, \mathscr{C}_{2}, \mathscr{C}_{3}, \ldots$ be a jointly independent sequence of fields of subsets of $X$. For each $n \geq 1$, the fields $\mathscr{C}_{1} \vee \mathscr{C}_{2} \vee \cdots \vee \mathscr{C}_{n}$ and $\mathscr{C}_{n+1}$ are independent.

Indication. This follows easily from the observation that sets in $\mathscr{C}_{1} \vee \mathscr{C}_{2} \vee \cdots \vee \mathscr{C}_{n}$ are unions of sets of the form $C_{1} \cap C_{2} \cap \cdots \cap C_{n}$ where $C_{i} \in \mathscr{C}_{i}$ for each $i$.

Lemma 1.2. Let $\mu: \mathscr{A} \rightarrow G$ and $\nu: \mathscr{B} \rightarrow G$ be $G$-valued charges with $\mu(X)=$ $\nu(X)$. If $\mathscr{A}$ and $\mathscr{B}$ are independent, then $\mu$ and $\nu$ have a common extension $\rho: \mathscr{A} \vee \mathscr{B} \rightarrow G$

Indication. This is Corollary 4.6 in [2].

We are now ready for our main result for independent fields. It will play an important role in the proof of Theorem 2.1 to come.

Theorem 1.3. Let $\tau_{k}: \mathscr{C}_{k} \rightarrow G(k=1,2, \ldots)$ be a sequence of $G$-valued charges, where $\left(\mathscr{C}_{k}\right)$ is a jointly independent sequence of fields of subsets of $X$. 
If $\tau_{1}(X)=\tau_{2}(X)=\cdots$, then there is a charge $\tau: \mathscr{P}(X) \rightarrow G$ such that, for each $k, \tau(C)=\tau_{k}(C)$ for all $C \in \mathscr{C}_{k}$.

Proof. We define a sequence of charges $\rho_{n}: \mathscr{C}_{1} \vee \mathscr{C}_{2} \vee \cdots \vee \mathscr{C}_{n} \rightarrow G$. Of course $\rho_{1}=\tau_{1}$. Assume that $\rho_{n}$ has been defined in such a way that $\rho_{n}$ is a common extension of $\tau_{1}, \tau_{2}, \ldots, \tau_{n}$. Apply Lemma 1.1 and 1.2 to obtain a common extension of $\rho_{n}: \mathscr{C}_{1} \vee \mathscr{C}_{2} \vee \cdots \vee \mathscr{C}_{n} \rightarrow G$ and $\tau_{n+1}: \mathscr{C}_{n+1} \rightarrow G$. Call this common extension $\rho_{n+1}: \mathscr{C}_{1} \vee \mathscr{C}_{2} \vee \cdots \vee \mathscr{C}_{n+1} \rightarrow G$.

Define $\mathscr{D}=\bigcup_{k} \mathscr{C}_{1} \vee \cdots \vee \mathscr{C}_{k}$. Then $\mathscr{D}$ is a field, and there is a charge $\tau: \mathscr{D} \rightarrow G$ such that $\tau=\rho_{n}$ on $\mathscr{C}_{1} \vee \cdots \vee \mathscr{C}_{n}$ for each $n$. By Lemma 0.1 , the domain of $\tau$ may be extended to all of $\mathscr{P}(X)$. This $\tau$ is an extension of each $\tau_{n}$. Q.E.D.

\section{The Chinese ReMAinder PRoperty}

Our main theorem follows, to the effect that the common extension property implies the Chinese remainder property. Thus nontrivial free Abelian groups, in particular $\mathbf{Z}$, cannot have the extension property. We have the following diagram of implications:

$$
\begin{aligned}
\text { divisible } & \Longrightarrow \text { algebraically compact } \\
& \Longrightarrow \text { common extension property } \Longrightarrow \text { Chinese remainder property }
\end{aligned}
$$

Theorem 2.1. Let $G$ be an Abelian group with the common extension property. Then $G$ has the Chinese remainder property.

Remark. Actually we show that $G$ has the Chinese remainder property so long as every consistent pair $\mu, \nu$ with $\nu=0$ has a common extension. But, really, it is not too hard to show that $G$ has the common extension property if and only if any two such $\mu$ and $\nu$ can be extended.

Proof. Define $X=\{0,1,2, \ldots\}$ and let $\mathscr{A}$ [resp. $\mathscr{B}$ ] be the field of all subsets of $X$ that are unions of the sets $\{0\},\{1,2\}, \ldots,\{2 m-1,2 m\}, \ldots$ [resp. the sets $\{0,1\},\{1,2\}, \ldots,\{2 m, 2 m+1\}, \ldots]$. Notice that $\mathscr{A} \cap \mathscr{B}$ is the trivial field $\{\varnothing, X\}$, so that $G$-valued charges $\mu: \mathscr{A} \rightarrow G$ and $\nu: \mathscr{B} \rightarrow G$ are consistent if and only if $\mu(X)=\nu(X)$. We define $\nu=0$, the identically zero charge on $\mathscr{B}$.

Suppose now that $a_{1}, a_{2}, \ldots$ are elements of $G$ and that $n_{1}, n_{2}, \ldots$ is a sequence of pairwise relatively prime, positive integers. Since congruences of the form $x \equiv a(\bmod 1)$ are trivial, we may assume that $n_{k} \geq 2$ for each $k$.

The basic idea of the proof is this: We construct $\mu$ and $\nu$ and functions $f, g_{k}, l_{k}$, and $h_{k}$ such that for each $k, f-g_{k}=k \cdot l_{k}+h_{k}$ and such that for any common extension $\rho$ of $\mu$ and $\nu$, we have $\int g_{n_{k}} d \rho=a_{k}$ and $\int h_{n_{k}} d \rho=0$. Then

$$
\begin{aligned}
\int f d \rho-\int g_{n_{k}} d \rho & =n_{k} \int l_{n_{k}} d \rho+\int h_{n_{k}} d \rho, \\
\int f d \rho-a_{k} & =n_{k} \int l_{n_{k}} d \rho .
\end{aligned}
$$


Thus the element $x=\int f d \rho$ in $G$ will be a solution of the sequence of congruences $x \equiv a_{k}\left(\bmod n_{k}\right)$.

For $k \geq 1$ and $0 \leq i \leq k-1$, define the sets

$$
A_{i}^{k}=\{m \in X: m \equiv i(\bmod k)\} .
$$

Then the sets $A_{0}^{k}, A_{1}^{k}, \ldots, A_{k-1}^{k}$ form a partition of $X$ into $k$ nonempty sets. Let $\mathscr{C}_{k}$ be the field on $X$ generated by this partition.

Claim. The sequence of fields $\mathscr{C}_{n_{1}}, \mathscr{C}_{n_{2}}, \ldots$ is jointly independent.

Proof of Claim. For each $k$ and every set of integers $i_{1}, i_{2}, \ldots, i_{k}$ with $0 \leq$ $i_{j} \leq n_{j}-1$, we have $A_{i_{1}}^{n_{1}} \cap A_{i_{2}}^{n_{2}} \cap \cdots \cap A_{i_{k}}^{n_{k}} \neq \varnothing$. This is simply a restatement of the classical Chinese Remainder Theorem (0.2). The claim is thus established.

We now define a $G$-valued charge $\tau_{k}$ on $\mathscr{C}_{n_{k}}\left(n_{k} \neq 2\right)$ by setting

$$
\begin{gathered}
\tau_{k}\left(A_{1}^{n_{k}}\right)=a_{k}, \quad \tau_{k}\left(A_{2}^{n_{k}}\right)=-a_{k}, \\
\tau_{k}\left(A_{i}^{n_{k}}\right)=0 \quad\left(i=0 \text { and } 3 \leq i \leq n_{k}-1\right) .
\end{gathered}
$$

If $n_{k}=2$, put

$$
\tau_{k}\left(A_{1}^{2}\right)=a_{k}, \quad \tau_{k}\left(A_{0}^{2}\right)=-a_{k}
$$

Note that $\tau_{k}(X)=0$ for each $k$. It follows from Theorem 1.3 that there is a charge $\tau: \mathscr{P}(X) \rightarrow G$ serving as a common extension for the charges $\tau_{k}$. Define $F: X \rightarrow X$ by $F(m)=2 m$ and let $\mu$ be the image charge under the action of $F$, viz. $\mu(A)=\tau\left(F^{-1}(A)\right)$ for each $A \in \mathscr{A}$. Note that $\mu(X)=0$. If $G$ indeed has the common extension property, then there is a $G$-valued charge $\rho: \mathscr{A} \vee \mathscr{B} \rightarrow G$ extending both $\mu$ and $\nu$.

We now define the functions $f, g_{k}, l_{k}$, and $h_{k}$ on $X$ for $k=2,3, \ldots$ as follows. If $m$ and $k$ are integers with $k \geq 2$, let $[m]_{k}$ be the unique integer such that $0 \leq[m]_{k} \leq k-1$ and $m \equiv[m]_{k}(\bmod k)$. Thus $[m]_{k}$ is $m$ "reduced" modulo $k$. Then put

$$
\begin{gathered}
f(m)=[m-1]_{2} \quad g_{k}(2 m-1)=g_{k}(2 m)=-[m-1]_{k} \\
h_{k}(2 m)=h_{k}(2 m+1)=[m]_{k} \quad l_{k}(m)= \begin{cases}1 & \text { if } 2 k \mid m \\
0 & \text { if } 2 k \not m\end{cases}
\end{gathered}
$$

As an example, we display some of the values for the case $k=4$.

\begin{tabular}{c|rrrrrrrrrrrrr}
$m$ & 0 & 1 & 2 & 3 & 4 & 5 & 6 & 7 & 8 & 9 & 10 & 11 & 12 \\
\hline$f(m)$ & 1 & 0 & 1 & 0 & 1 & 0 & 1 & 0 & 1 & 0 & 1 & 0 & 1 \\
$g_{4}(m)$ & -3 & 0 & 0 & -1 & -1 & -2 & -2 & -3 & -3 & 0 & 0 & -1 & -1 \\
$h_{4}(m)$ & 0 & 0 & 1 & 1 & 2 & 2 & 3 & 3 & 0 & 0 & 1 & 1 & 2 \\
$l_{4}(m)$ & 1 & 0 & 0 & 0 & 0 & 0 & 0 & 0 & 1 & 0 & 0 & 0 & 0
\end{tabular}


It is clear that for each $k, g_{k} \in S(X, \mathscr{A})$ and $h_{k} \in S(X, \mathscr{B})$. Although somewhat tedious, simple case-checking proves the identity

$$
f-g_{k}=k l_{k}+h_{k} \quad(k \geq 2) .
$$

Put $B_{i}^{k}=\left\{2 m, 2 m-1: m \in A_{i}^{k}\right\}$ for $0 \leq i \leq n_{i}-1$. Then $F^{-1}\left(B_{i}^{k}\right)=A_{i}^{k}$. Note that $g_{k}$ is constant on the the sets $B_{0}^{k}, B_{1}^{k}, \ldots, B_{k-1}^{k}$ and that

$$
g_{k}=0 \quad \text { on } B_{1}^{k} \quad g_{k}=-1 \quad \text { on } B_{2}^{k} .
$$

We calculate for the case $n_{k} \neq 2$ :

$$
\begin{aligned}
\mu\left(B_{i}^{n_{k}}\right) & =\tau\left(F^{-1}\left(B_{i}^{n_{k}}\right)\right)=\tau\left(A_{i}^{n_{k}}\right) \\
& =\tau_{k}\left(A_{i}^{n_{k}}\right)= \begin{cases}a_{k} & i=1 \\
-a_{k} & i=2 \\
0 & i=0 \text { and } 3 \leq i \leq k-1 .\end{cases}
\end{aligned}
$$

For the case $n_{k}=2$, we have

$$
\mu\left(B_{0}^{2}\right)=-a_{k} \quad \mu\left(B_{1}^{2}\right)=a_{k} .
$$

We now apply $(* *)$ and $(* * *)$ to obtain $\left(n_{k} \neq 2\right)$

$$
\begin{aligned}
\int g_{n_{k}} d \rho=\int g_{n_{k}} d \mu & =\int_{B_{0}^{n_{k}}} g_{n_{k}} d \mu+\int_{B_{1}^{n_{k}}} g_{n_{k}} d \mu+\cdots+\int_{B_{n_{k}-1}^{n_{k}}} g_{n_{k}} d \mu \\
& =0+0-\mu\left(B_{2}^{n_{k}}\right)+0+\cdots+0 \\
& =-\left(-a_{k}\right)=a_{k} .
\end{aligned}
$$

If $n_{k}=2$, the same result obtains. Integration of $(*)$ against $\rho$ yields

$$
\int f d \rho-\int g_{n_{k}} d \rho=n_{k} \int l_{n_{k}} d \rho+\int h_{n_{k}} d \rho .
$$

We have $\int h_{n_{k}} d \rho=\int h_{n_{k}} d \nu=0$, so that for each $k=1,2, \ldots$

$$
\int f d \rho-a_{k}=n_{k} \int l_{n_{k}} d \rho \text {. }
$$

Thus we see that the element $x=\int f d \rho$ in $G$ is a solution of the congruences $x \equiv a_{k}\left(\bmod n_{k}\right)$ for $k=1,2, \ldots$ We have established that $G$ has the Chinese remainder property. Q.E.D.

Corollary 2.2. The group $\mathbf{Z}$ does not have the common extension property. The same is true for any group in which there is an infinite cyclic summand.

Corollary 2.3. There are two 0-1 valued charges with no common integer-valued extension.

Indication. Let $n_{1}=2, n_{2}=3, n_{3}=5, \ldots$ be the sequence of all prime numbers. If, in the proof of Theorem 2.1, we set

$$
\tau_{k}\left(A_{i}^{n_{k}}\right)=\left\{\begin{array}{ll}
0, & i=1 \\
1, & i \neq 1
\end{array} \text { for } n_{k} \neq 2\right.
$$


and

$$
\tau_{k}\left(A_{i}^{2}\right)=\left\{\begin{array}{ll}
0, & i=0 \\
1, & i=1
\end{array} \text { for } n_{1}=2,\right.
$$

then, with extra care, the resulting $\mu$ becomes a $0-1$ valued charge on $\mathscr{A}$. Take $\nu$ on $\mathscr{B}$ to be the point mass at 0 . Then $\mu$ and $\nu$ are $0-1$ valued charges not having a common integer-valued extension.

\section{COMMON EXTENSIONS AND COTORSION GROUPS} $F$.

A group $G$ is a cotorsion group if $\operatorname{Ext}(F, G)=0$ for each torsion-free group

Lemma 3.1. Let $G$ be an Abelian group. The following conditions are equivalent:

(1) $G$ is a cotorsion group.

(2) $G$ is the epimorphic image of an algebraically compact group.

(3) $\operatorname{Ext}(D, G)=0$ for some nonzero torsion-free divisible group $D$.

Indication. See $\S 54$ of [4], especially Proposition 54.1.

The cotorsion property is thus somewhat weaker than algebraic compactness. In fact, it provides a characterisation of groups with the common extension property.

Theorem 3.2. An Abelian group $G$ has the common extension property if and only if $G$ is a cotorsion group.

Proof. Suppose that $G$ is a cotorsion group. By Lemma 3.1, there is an algebraically compact group $K$ and an epimorphism $\phi: K \rightarrow G$. Let $\mu: \mathscr{A} \rightarrow G$ and $\nu: \mathscr{B} \rightarrow G$ be two consistent $G$-valued charges. Let $\psi_{\mu}$ and $\psi_{\nu}$ be the $G$-valued group homomorphisms induced by $\mu$ and $\nu$ on $S(X, \mathscr{A})$ and $S(X, \mathscr{B})$, respectively:

$$
\psi_{\mu}(f)=\int f d \mu, \quad \psi_{\nu}(f)=\int f d \nu
$$

Now $S(X, \mathscr{A}), S(X, \mathscr{B})$, and $S(X, \mathscr{A} \cap \mathscr{B})$ are free Abelian groups [2, Theorem 1.5]. Moreover, we can find bases $B_{\mathscr{A}}$ for $S(X, \mathscr{A})$ and $B_{\mathscr{B}}$ for $S(X, \mathscr{B})$ such that $B_{\mathscr{A}} \cap B_{\mathscr{B}}$ is a basis for $S(X, \mathscr{A} \cap \mathscr{B})$. (See [2].) We shall obtain $\overline{\psi_{\mu}}: S(X, \mathscr{A}) \rightarrow K$ and $\overline{\psi_{\nu}}: S(X, \mathscr{B}) \rightarrow K$ such that $\overline{\psi_{\mu}}$ and $\overline{\psi_{\nu}}$ are consistent (i.e. they agree on $S(X, \mathscr{A} \cap \mathscr{B})$ ).

For $f \in B_{\mathscr{A}} \cap B_{\mathscr{B}}$, define $\overline{\psi_{\mu}}(f)=\overline{\psi_{\nu}}(f)$ to be any element of $\phi^{-1}\left(\psi_{\mu}(f)\right)=$ $\phi^{-1}\left(\psi_{\nu}(f)\right)$. For $f \in B_{\mathscr{A}}-B_{\mathscr{B}}$, define $\overline{\psi_{\mu}}(f)$ to be any element of $\phi^{-1}\left(\psi_{\mu}(f)\right)$. For $f \in B_{\mathscr{B}}-B_{\mathscr{A}}$, define $\overline{\psi_{\nu}}(f)$ to be any element of $\phi^{-1}\left(\psi_{\nu}(f)\right)$. Then $\overline{\psi_{\mu}}$ and $\overline{\psi_{\nu}}$ extend uniquely as homomorphisms on $S(X, \mathscr{A})$ and $S(X, \mathscr{B})$ agreeing on $S(X, \mathscr{A}) \cap S(X, \mathscr{B})=S(X, \mathscr{A} \cap \mathscr{B})$. Then the set functions $\bar{\mu}: \mathscr{A} \rightarrow K$ and $\bar{\nu}: \mathscr{B} \rightarrow K$ defined by $\bar{\mu}(A)=\overline{\psi_{\mu}}\left(I_{A}\right)$ and $\bar{\nu}(B)=\overline{\psi_{\nu}}\left(I_{B}\right)$ are consistent $K$-valued charges. Since $K$ is algebraically compact, it has the common extension property (0.5). Let $\bar{\rho}$ be a common extension of $\bar{\mu}$ and $\bar{\nu}$. Then $\rho=\phi \circ \bar{\rho}$ is a common extension for $\mu$ and $\nu$. 
Now suppose that $G$ has the common extension property. Let $X, \mathscr{A}$, $\mathscr{B}$ be as in the proof of Theorem 2.1. Put $A=S(X, \mathscr{A} \vee \mathscr{B})$ and $A^{\prime}=$ $S(X, \mathscr{A})+S(X, \mathscr{B}) \subseteq A$. Consider the short exact sequence:

$$
0 \rightarrow A^{\prime} \stackrel{j}{\rightarrow} A \rightarrow A / A^{\prime} \rightarrow 0
$$

where $j$ is the inclusion map. Since $A$ and $A^{\prime}$ are free groups, Theorem 51.3 in [4] yields a dual exact sequence:

$$
0 \leftarrow \operatorname{Ext}\left(A / A^{\prime}, G\right) \leftarrow \operatorname{Hom}\left(A^{\prime}, G\right) \stackrel{j^{*}}{\leftarrow} \operatorname{Hom}(A, G) \leftarrow \operatorname{Hom}\left(A / A^{\prime}, G\right) \leftarrow 0 .
$$

Since $\mathrm{G}$ has the common extension property, $j^{*}$ is a surjection. Thus $\operatorname{Ext}\left(A / A^{\prime}, G\right)=0$. Taking $f \in A$ from the proof of Theorem 2.1, we see that $f+A^{\prime}$ is an element of $A / A^{\prime}$ that is divisible by every nonzero integer.

Now $A^{\prime}$ is a pure subgroup of $A$. (See Example 2.4 in [2], where the same groups are used. In fact, one can show that $S(X, \mathscr{C})+S(X, \mathscr{D})$ is a pure subgroup of $S(X, \mathscr{C} \vee \mathscr{D})$ for any fields $\mathscr{C}$ and $\mathscr{D}$.) It follows that $A / A^{\prime}$ is torsion free. Thus $A / A^{\prime}$ contains a nontrivial divisible subgroup $D$, so that $A / A^{\prime}=D \oplus R$. So $0=\operatorname{Ext}\left(A / A^{\prime}, G\right)=\operatorname{Ext}(D, G) \oplus \operatorname{Ext}(R, G)$. Thus $\operatorname{Ext}(D, G)=0:$ Lemma 3.1 implies that $G$ is a cotorsion group. Q.E.D.

Note. The statement of Theorem 3.2 was told to us by K. M. Rangaswamy and J. D. Reid. The proof given above was discovered shortly afterwards by the authors.

\section{ACKNOWLEDGMENT}

The authors wish to thank G. Bergman for some extremely enlightening correspondence as well as A. Molitor for his willingness to check some of our work.

\section{REFERENCES}

1. A. Basile and K. P. S. Bhaskara Rao, Common extensions of group-valued charges, preprint.

2. K. P. S. Bhaskara Rao and R. M. Shortt, Common extensions for homomorphisms and groups-valued charges, Rend. Circ. Mat. (Palermo) (to appear).

3. T. Carlson and K. Prikry, Ranges of signed measures, Period. Math. Hungar. 13 (1982) 151-155.

4. L. Fuchs, Infinite Abelian groups, Vol. I, Academic Press, New York, 1970.

5. I. Niven and H. S. Zuckerman, An introduction to the theory of numbers, 3rd ed., Wiley, New York, 1972.

Department of Mathematics, Wesleyan University, Middletown, Connecticut 06457

Department of Mathematics, Indian Statistical Institute, Bangalore, India 